\title{
ARTIGO 9
}

\section{COMPORTAMENTO INFORMACIONAL DE GESTORES DE UMA SUPERINTENDÊNCIA REGIONAL DE SAÚDE}

Tamires Rezende Passos ${ }^{1}$, Wanesia Saldanha Costa ${ }^{1}$, Jessica Silva Oliveira ${ }^{1}$, Humberto Ferreira de Oliveira Quites ${ }^{2}$, Regina Consolação dos Santos ${ }^{3}$, Ricardo Bezerra Cavalcante ${ }^{2}$

Objetivo: compreender o comportamento informacional de gestores em uma Superintendência Regional de Saúde. Metodologia: estudo de caso, de abordagem qualitativa, que utilizou como referencial teórico o comportamento informacional de Wilson. Realizaram-se entrevistas com gestores de uma superintendência em Minas Gerais, analisadas a partir da Análise de Conteúdo. Resultados: as necessidades e busca por informações estavam atreladas às demandas de soluções emergenciais. Dentre as várias fontes, as pessoas foram reconhecidas em um fluxo informacional verticalizado. Informações de baixa qualidade foram utilizadas, gerando incerteza para as decisões. Conclusão: o comportamento informacional dos gestores é assistemático e possui fragilidades que podem dificultar o planejamento e a tomada de decisões assertivas.

Descritores: Comportamento de Busca de Informação, Gestão da Informação em Saúde, Gestão em Saúde.

\section{INFORMATIONAL BEHAVIOR OF MANAGERS IN REGIONAL SUPERINTENDENCE OF HEALTH}

Objective: to understand the informational behavior of managers in a regional superintendence of health. Methodology: Case study with qualitative approach that used Wilson behavior as a theoretical reference. Interviews were conducted with managers of a superintendency in Minas Gerais, and analyzed from the Content Analysis. Results: the necessities and pursuit for information was linked to the demands of emergency solutions. Among the various sources, people were recognized in a vertical information flow. Low quality of information were used generating uncertainty in decisions. We conclude that the informational behavior of managers is unsystematic and potentially damaging to the management of information.

Descriptors: Search Behavior Information, Health Information Management, Health Management.

\section{COMPORTAMIENTO INFORMATIVO DE GERENTES DE UNA SUPERINTEDENCIA REGIONAL DE SALUD}

Objetivo: Estudio de caso con enfoque cualitativo. Buscado entender el comportamiento informativo de los gestores en Salud. Utilizamos la teoría del comportamiento de información de Wilson. Entrevistamos a los gestores de una superintendencia de salud en Minas Gerais. Se utilizó el Análisis de Contenido para analizar los datos. Las necesidades y búsqueda de información estaba vinculada a las demandas de soluciones emergenciales. Las personas fueron reconocidas como fuentes en un flujo de información vertical. Hubo baja calidad de la información que genera decisiones inciertas. Se concluye que el comportamiento de información de los gestores no es sistemática y potencialmente dañino para la gestión de la información.

Descriptores: Comportamiento de Búsqueda de Información, Gestión de Información Sanitaria, Gestión de la Salud.

'Enfermeira. Membro do Nucleo de Estudos e Pesquisas em Avaliação da Universidade Federal de São João Del Rei - NEPAG-UFSJ.

${ }^{2}$ Enfermeiro. Doutor em Enfermagem. Membro do NEPAG-UFSJ. ricardocavalcante@ufsj.edu.br

${ }^{3}$ Enfermeira. Mestre em Biotecnologia. Membro do NEPAG-UFSJ) 


\section{INTRODUÇÃO}

O Ministério da Saúde, reconhecendo a necessidade de avançar no contexto da utilização da informação com vistas a uma gestão qualificada em saúde, propôs em sua agenda de prioridades de pesquisa, dentre outros, os temas: estudos sobre o uso da informação nos processos decisórios do Sistema Único de Saúde (SUS) e estudos voltados para a determinação das necessidades de informação(1).

O comportamento informacional é a totalidade do comportamento humano em relação às fontes e canais de informação, incluindo informação ativa e passiva, busca e uso da informação(2). Também se refere ao modo como os individuos lidam com a informação, incluindo a busca, o uso, a alteração, a troca, o acúmulo e, até mesmo, o ato de ignorar os informes ${ }^{(3,4)}$. Nessa ótica, o sujeito é quem determina sua relação com a informação, sendo influenciado por fatores como cultura, memória, educação e outros.

Estudos sobre comportamento informacional estão em desenvolvimento na área da saúde e demonstram a necessidade de práticas de gestão da informação mais sistemáticas e que proporcionem suporte à gestão dos serviços e à assistência a saúde(5-9). As necessidades informacionais dos profissionais estão relacionadas com suas práticas clínicas e demandas de programas governamentais, sendo suas fontes de informação, predominantemente, livros, sistemas de informação e opiniões de outros profissionais mais experientes.

Apesar da existência de alguns estudos sobre comportamento informacional, no contexto da saúde, as pesquisas são focadas nos profissionais de saúde em suas atividades assistenciais, sendo necessários estudos sobre o comportamento informacional de gestores, visto a sua importância em decisões estratégicas no contexto da organização de toda a rede de atenção em saúde. Contudo, surge o seguinte questionamento: como ocorre o comportamento informacional de gestores de uma Superintendência Regional de Saúde em relação ao seu cotidiano de trabalho? Assim, este estudo teve como objetivo compreender o comportamento informacional de gestores relacionado ao cotidiano de trabalho em uma Superintendência Regional de Saúde (SRS) do estado de Minas Gerais (MG).

\section{METODOLOGIA}

Trata-se de um estudo de caso, de abordagem qualitativa, norteado pelo referencial teórico de Wilson ${ }^{(2)}$ sobre comportamento informacional. O cenário escolhido intencionalmente foi a SRS responsável pela Região Ampliada Oeste de Saúde de Minas Gerais. A SRS tem, por finalidade, apoiar, implementar e monitorar as políticas e ações de saúde, fortalecendo a governança regional entre 54 municípios e a Secretaria de Estado de Saúde (SES). Foram convidados a participar da pesquisa todos os 12 (doze) profissionais da SRS que ocupam cargos de diretoria, superintendência e coordenadoria, sendo esses denominados gestores. Desses, apenas um recusou-se a participar da pesquisa.

A coleta de dados foi realizada no período de maio a julho de 2015 por meio de entrevistas semiestruturadas, contendo questões relacionadas ao comportamento informacional. Como uma segunda fonte de evidências, foi utilizada a Técnica de Incidente Crítico (TIC) descrita por Flanagan ${ }^{(10)}$ visando compreender o comportamento informacional dos gestores.

Os dados coletados foram organizados e analisados a partir da análise de conteúdo, modalidade temático-categorial(11). Foi realizada primeiramente a pré-análise a partir da leitura flutuante das entrevistas. Posteriormente, as unidades de registro foram codificadas e segregadas formando os núcleos de sentido. Em um terceiro momento, os núcleos de sentido foram agrupados em categorias que os representavam.

Este estudo obedeceu aos princípios éticos da Resolução 466/2012 do Conselho Nacional de Saúde, e foi aprovado por Comitê de Ética em Pesquisa, segundo parecer número $578.073 / 2014$

\section{RESULTADOS}

\section{Necessidades, busca e fontes de informações}

Inicialmente percebemos que as necessidades de informações estão atreladas à busca por resolução de problemas que emergem no trabalho. (...) De acordo com as 
dificuldades que se apresentam no meu dia-dia de trabalho, é que eu busco de fato, pra achar solução (...) (EI).

Nesse contexto, independente do grau de dificuldade, há uma busca pelo entendimento do problema, partindo daí a busca por informações que possam facilitar a compreensão e responder aos questionamentos, como, por exemplo, o surgimento de uma doença: (...) Como que essa doença chegou aqui? Ela não tem costume de tá aqui, então como ela chegou aqui?(E8).

Dentre as fontes de informação utilizadas pelos gestores em suas atividades, destacam-se as normas legislativas, necessárias para a realização do cotidiano de trabalho (...) a legislação do Ministério da Saúde, quanto à legislação do estado, então é a legislação de forma geral, ela éfundamental. (E10); (...) É que a gente trabalha em cima de legislação então a gente tem que ficar atenta a essas informações. (E5)

As pessoas, apesar de não serem muitas vezes reconhecidas como fontes oficiais de informações, são legitimadas pelos profissionais que fazem parte do fluxo informacional: (...) os profissionais dos municipios, que também é uma fonte de informação, embora ela não seja oficial, mas ela é uma fonte de informação importante por que, é quando as pessoas trazem informações. (El)

Outra fonte reconhecida pelos gestores são os Sistemas de Informação em Saúde (SIS). Alguns gestores entendem que os SIS possuem informações consistentes e são indispensáveis ao cotidiano de trabalho: (...) Hoje a fonte mais consistente são os sistemas oficiais de informação. Não diria que ela é a mais importante, diria que ela é mais consistente. Pra tomada de decisão sem dúvida nenhuma é o que a gente utiliza (EI).

As notificações também emergiram como fonte de informação. (...) Com maior frequência é a notificação de agravos. A gente monitora toda semana, enquanto os agravos são controlados. (E8); (...) Hoje a nossa principal informação é a notificação de risco. (E3). (...) informações de jornais, revistas, imprensa (EI); (...) pela internet. Têm fóruns de discussão, tem o email institucional. (E2).

\section{Avaliação da qualidade das informações}

Não há um processo ou uma rotina explícita de avaliação da qualidade das informações. Os gestores acreditam que
- conhecimento prático adquirido por suas experiências anteriores são capazes de respaldar a avaliação da qualidade da informação coletada. (...) A gente avalia com base no conhecimento prático que a gente já tem. E a gente tem condição de fazer uma crítica se aquela informação, ela tá correta ou não. (El). Entretanto, reconhecem essa situação como uma dificuldade que desqualifica a informação: (...) A dificuldade que a gente tem com relação à informação é da qualidade da informação na ponta (El).

O parâmetro utilizado sobre a qualidade das informações é baseado no contexto dos acontecimentos e dependente das pessoas que criam esses parâmetros: (...) Se tá numa epidemia, a gente sabe que a notificação está sendo pequena, não tá sendo total. Entendeu? A gente já espera que tenha sido, é esquecido por aí alguns casos(E8).

Mesmo reconhecendo a baixa qualidade das informações, os gestores dão sequência na sua utilização e assumem o risco de sua infidedignidade: (...) Eu tenho que acreditar no técnico, eu tenho que confiar na equipe. Então vai no próprio senso do risco que ele teve de trazer pra mim e diante do que ele traz eu tenho que tomar decisão (E3).

O fluxo de informações entre
as esferas de gestão
Os gestores enfatizaram
a existência de um fluxo
vertical de informações
que tem origem na SRS
para SES, onde as trocas
de informações acontecem por serem normatizadas: (...) Nós temos que encaminhar para o nivel central (SES), os relatórios, o consolidado da situação de aprovação do relatório anual de gestão, do plano municipal de saúde e outras informações que eles nos demandam. (E6).

Há também um fluxo de informações entre a SRS e os municípios descrita como casual em função da necessidade de consulta ou demanda: (...)em alguma situação especifica, eles perguntam: olha a gente precisa saber isso. Ai a gente compartilha a informação, né?! (El); (...)a gente faz visita no município, faz orientação direta no local, nas equipes, onde é solicitado (E10).

Entretanto, outros relatos sugerem inconsistência desse fluxo de informações: (...)É muito fraca minha interação junto, por exemplo, com a atenção a saúde, com atenção primária, com a gestão micro(...) (E3); (...) não é o meu 
trabalho compartilhar! E pra que? Se vierem me perguntar eu compartilho ...(E8).

\section{Utilização das informações e a tomada de decisões}

De forma geral, as informações divulgadas a partir de portarias ministeriais e de programas governamentais são responsáveis por direcionar as atividades da SRS e também dos municípios. (...) a gente utiliza informações pra todas as decisões que a gente vai tomar em relação ao monitoramento ou a realização do apoio técnico, são orientações. (E8).

Há também a dependência dos gestores da SRS de informações (autorizações) centralizadas na SES. (...) A gente demanda da secretaria(SES) o tempo inteiro de autorizações até mesmo para tomada de ações. (E4). Nesse contexto, há o anseio de que as decisões definidas pela SES sejam cumpridas, e isso dispara um processo de responsabilização pelo cumprimento da decisão:

(...) Porque se há mudanças e se você não toma frente, as mudanças não acontecem, aí você não corresponde aos anseios que o nivel central espera (SES). (E5).

Além disso, algumas informações provenientes da SES não são discutidas, sendo informadas e impostas cabendo o seu cumprimento na SRS: (...) os terceirizados daqui não registravam pontos, (...) ai veio a informação que a partir da data tal, todos passariam a fazer o registro eletrônico! Então você tem que tomar atitude, sair e dizer: ó pessoal agora vai ser assim (...) (E5).

As decisões também são disparadas a partir de um problema verificado, conformando-se como uma informação que se desvia de um padrão de normalidade: (...) É necessário fazer uma visita naquele municipio (...) pra ver qual é a necessidade dele, qual é a dificuldade, o que está acontecendo (...) Com base na informação que eu receber tomo a decisão (E10).

Apesar de sugerir uma sistemática de utilização de informações para a tomada de decisão, reconhecem-se decisões emergenciais e sem planejamento: (...)Eu fico apagando fogo toda hora (...) Não tem uma estratégia a longo prazo, chegou? Apaga! Todas! Porque como é denúncia e todas são prioridades, não tem como planejar! (E3)

\section{DISCUSSÃO}

Os achados referentes às necessidades e buscas de informação pelos gestores apontam, prioritariamente, para a necessidade de suprir demandas emergenciais, de repasses financeiros, as pactuações intergestoras e ao cumprimento de metas da SES. Assim, não estão definidas as necessidades informacionais prioritárias na SRS, a fim de subsidiar as práticas de trabalho local desses gestores. Quando não há a determinação das necessidades informacionais especificas, a tendência é que todo o comportamento informacional fique assistemático, podendo comprometer a qualidade do serviço prestado $^{(2,4)}$

Foram reconhecidas várias fontes de informações utilizadas pelos gestores, entretanto, é importante salientar que a simples existência dessas fontes e seu acesso não garantem o uso adequado dessas informações ${ }^{(12)}$. De forma geral, as informações precisam ser transmitidas a pessoas certas, de uma forma que despertem atenção, sejam compreendidas e com possibilidade de uso(4).

Outro aspecto relacionado a essa temática foi a dependência das pessoas como fontes de informações. Verificou-se que há um conhecimento tácito emanado dessas pessoas que auxilia no cotidiano de trabalho. Este é considerado importante para as organizações, sendo originado das experiências pessoais, dos debates, das opiniões, dos valores individuais advindos de um contexto próprio. Porém, é difícil de ser articulado em uma linguagem formal e de complexa administração(13,14), como a gestão burocratizada da SRS, justificada pela necessidade de controle, estabilidade e coesão, o que pode desencadear prejuizos nesse tipo de conhecimento ${ }^{(14)}$

Não há um cuidado explícito quanto à avaliação e à qualidade das informações desenvolvidas. Tal prática reforça a opção por um processo assistemático de gestão da informação, que desfavorece o ambiente organizacional(2), inviabilizando o uso da informação como um recurso favorável ao planejamento e a tomada de decisões assertivas ${ }^{(1)}$.

Contudo, as informações mais utilizadas seguem um fluxo vertical: o ministério define uma norma a ser cumprida no estado e municípios, uma normativa legitimada a priori. 
Esse processo verticalizado e burocratizado das informações submete profissionais e gestores no lócus assistencial a coletas de dados e elaboração de relatórios/consolidados sem sentido e com pouca aplicabilidade(15). É importante destacar que tal fluxo tem gerado um compartilhamento de informações incipiente, tendo em vista que a troca de informações é involuntária ${ }^{(2)}$. Há também a incompreensão da necessidade de compartilhamento das informações, falta de afinidade entre algumas pessoas, entre outros aspectos que obstaculizam o fluxo de informações. Nesse contexto, é essencial potencializar nas pessoas um comportamento informacional voltado para o compartilhamento de informações, bem como desenvolver ações que impactem nos fluxos informacionais ${ }^{(2)}$.

Corroborando com o exposto até aqui, a utilização das informações não favorece uma tomada de decisão baseada na deliberação e na análise prévia das informações. O caráter normativo burocrático inviabiliza o processo de análise, planejamento e, consequentemente, o distancia de decisões mais resolutivas.

\section{CONCLUSÃO}

No cenário analisado, o comportamento informacional é assistemático e possui fragilidades que podem dificultar o planejamento e a tomada de decisões assertivas. Não se estimula o compartilhamento de informações e nem a avaliação de sua qualidade, o que gera incertezas no trato com a informação. Considera-se que este estudo não permite generalizações, sendo aplicável ao cenário e gestores participantes.

\section{REFERÊNCIAS}

1. Brasil. Ministério da Saúde. Agenda nacional de prioridades de pesquisa em saúde/Ministério da Saúde. Secretaria de Ciência, Tecnologia e Insumos Estratégicos. Departamento de Ciência e Tecnologia. Brasilia: Ministério da Saúde; 2011.

2. Wilson TD. Human Information Behavior. Special Issue on Information Science Research. 2000; 3(2): 49-55.

3. Nan Xiao, Raj Sharman, H.R. Rao, Shambhu Upadhyaya. Factors influencing online health information search: An empirical analysis of a national cancer-related survey. Decision Support Systems 2014 Jan; 57: 417-27.

4. Davenport TH. Ecologia da informação: por que só a tecnologia não basta para o sucesso na era da informação. l.ed. São Paulo: Futura: 1998.

5. Nogueira GD; Neves JTR. Estratégia Para a Gestão da Informação no Programa Saúde da Familia do Governo Brasileiro. Estratégia e Negócios 2009; 2(1):1-24.

6. Dickmann, P; Wittgens, K; Keeping, S; Mischler, D; Heudorf, U. Rethinking risk communication: information needs of patients, health professionals and the public regarding MRSA--the communicative behaviour of a public health network in Germany responding to the demand for information. Public Health 2016 Feb; 131:56-62.

7. Durieux, Nancy; Pasleau, Françoise; Piazza, Aurelie; Donneau, AnneFrançoise; Vandenput, Sandrina; Maillart, Christelle. Information behaviour of French-speaking speech-language therapists in Belgium: results of a questionnaire survey. Health Info Libr J 2016 Mar; 33(1): 61-76.

8. Tabosa HR, Pinto VB. Caracterização do comportamento de busca e uso de informação na área da saúde: o modelo de Ellis aplicado ao estudo do comportamento informacional de pacientes. Inf. \& Soc 2016 Mai/ Ago: 26(2): 225-38.

9. Wellichan DSP. Comportamento informacional de profissionais no domínio da saúde: um estudo junto ao Hospital de Reabilitação de Anomalias Craniofaciais da Universidade de São Paulo. Dissertação [mestrado] - Universidade Estadual Paulista Julio de Mesquita Filho. Faculdade de Filosofia e Ciências; 2015

10. Flanagan, JC. The critical incident technique. The Psychological Bulletin 1954; 51(4):327-58.

11. Bardin L. Análise de conteúdo. 70. ed. Lisboa: Ltda; 2015.

12. Samuel, Senait; Bayissa, Getachew; Asaminewu, Selam; Alaro, Tesfamichael. Electronic Information Sources Access and Use for Healthcare Services in Governmental and Non-Governmental Hospitals of Western Oromia, Ethiopia: A Cross Sectional Study. Ethiop J Health Sci 2016 Jul; 26(4): 341-50.

13. Becerril-Montekio, Victor; Alcalde-Rabanal, Jacqueline; Darney, Blair G; Orozco-Nuñez, Emanuel. Using systematized tacit knowledge to prioritize implementation challenges in existing maternal health programs: implications for the post MDG era. Health Policy Plan 2016 Oct; 31(8):1031-8.

14. Lemos B, Joia LA. Fatores relevantes à transferência de conhecimento tácito em organizações: um estudo exploratório. Gest. Prod 2012; 19(2):233-46.

15. Cavalcante RB, Bernardes MFVG, Gontijo TL, Guimarães EAA Oliveira VC. Sistema de informação da atenção básica: potencialidades e subutilização no processo decisório. Cogitare enferm[periódico na Internet]. 2013 Set [citado 2016 Nov 07] : 18( 3 ): 460-467. Disponivel em: http://www.revenf.bvs.br/scielo.php?script=sci_ arttext\&pid=S1414-85362013000300006\&lng=pt. 